\title{
Radix Entomolaris
}

Carlos Filloy Esna DDS 1

1. Profesor Facultad de Odontología, Universidad de Costa Rica, Costa Rica.

Autor para correspondencia: Dr. Carlos Filloy Esna - cfilloy@gmail.com

Recibido: 12-IX-2015

Aceptado: 16-XI-2015

Publicado Online First: 20-XI-2015

DOI: http://dx.doi.org/10.15517/ijds.v0i0.22144

\section{RESUMEN}

El conocimiento de la morfología y posibles variaciones anatómicas de los conductos radiculares es un requisito básico para el éxito del tratamiento endodóntico. Las molares inferiores pueden presentar una tercera raíz, la cual cuando se encuentra en dirección distolingual se denomina como radix entomolaris, y cuando ésta se encuentra en la superficie mesiovestibular, la anomalía se denomina radix paramolaris. El presente relato de caso clínico reporta el tratamiento endodóntico y consideraciones que el clínico debe conocer para el retratamiento de una primera molar inferior - radix entomolaris.

\section{PALABRAS CLAVE}

Endodoncia, Morfologia del conducto Radicular, Radix Entomolaris.

\section{ABSTRACT}

A comprehensive knowledge of the root canal morphology and its variations is a basic requisite for the success of the endodontic treatment. Mandibular molars may present a third or additional root, which if located lingually is called radix entomolaris or bucally is referred as radix paramolaris. This case report shows an endodontic retreatment of a radix entomolaris and details some variations in the approach to guarantee a successful terapy.

\section{KEYWORDS}

Endodontics, Root Canal Anatomy, Radix Entomolaris.

FILLOY C., 2015: Radix Entomolaris.-ODOVTOS-Int. J. Dental Sc., 17-3 (September-December): 49-54. 


\section{INTRODUCCIÓN}

El éxito de la terapia endodóntica depende en gran parte de la completa limpieza, desinfección y conformación de los sistemas de conductos radiculares, además del correcto selle tridimensional logrado con la obturación tridimensional y la posterior restauración coronaria definitiva (1). De esta forma, el conocimiento de la morfología del sistema de conductos radiculares, así como sus variaciones son aspectos fundamentales para el éxito del tratamiento endodóntico (1).

Usualmente el primer molar mandibular presenta 2 raíces y de 3-4 conductos radiculares (1), sin embargo en algunas ocasiones puede mostrar variaciones anatómicas. El número de raíces puede variar en los molares inferiores, en algunas ocasiones puede observarse la presencia de una tercera raíz, esta anomalía fue relatada en primer lugar en la literatura por Carabelli desde 1844 (2) y la llamó radix entomolaris. Esta raíz supernumerario se encuentra en dirección distolingual, principalmente en los primeros molares inferiores. Cuando se encuentra en la superficie mesiovestibular, la anomalía se denomina radix paramolaris. A pesar de que las causas de su formación han sido históricamente relacionadas con elementos genéticos, raza y factores externos que afectan a la odontogénesis, su etiología aún no ha sido definida con precisión. Esta anomalía es considerada de rara ocurrencia en individuos de raza caucásica, pero considerada una variación morfológica relativamente normal en individuos de grupos etnicos asiáticos, esquimales e indios americanos (3). La prevalencia de radix entomolaris oscila para la población africana en menos de un $3 \%$, euroasiáticos e indios en un 5 $\%$, europeos en menos del $4.2 \%$, mientras que en individuos con rasgos faciales mongoloides en rangos que van desde el $5 \%$ hasta el $30 \%$.47). Los Radix Entomolaris pueden estar presentes tanto en primeras, terceras molares, según la literatura se relata con menor frecuencia en las segundas molares mandibulares (6). Así mismo, existe entre un 50 y $60 \%$ de probabilidad que aparezcan bilateralmente.

A pesar de que los diferentes relatos de caso consideran escasa su presencia en la población caucásica, el conocimiento de su ocurrencia y manejo endodóntico es de fundamental importancia. En caso de requerir una terapia endodóntica, el correcto diagnostico de esta condición puede evitar complicaciones derivadas de un conducto no localizado, y por lo tanto no instrumentado ni obturado. De esta forma, el objetivo de este artículo es describir un relato de caso clínico de un retratamiento en un primer molar inferior con tres raíces y cuatro conductos radicualres (mesiovestibular, mesiolingual, distobucal, y centrolingual (radix entomolaris).

\section{REPORTE DE CASO}

Paciente de sexo masculino de 39 años de edad, raza blanca, cuya queja principal fue por dolor durante la masticación en la zona molar izquierda se presenta a consulta odontológica. El paciente no presenta ningún tipo de antecedentes médicos. Clínicamente se observa la pieza 3.6 con corona metal-porcelana y la pieza 3.7 sin ninguna restauración 0 caries. La pieza 3.7 presenta condiciones clínicas normales a las diferentes pruebas al frio, calor, pulpovitalometro, percusión o palpación. La pieza 3.6 no presento respuesta al frio, calor o pulpovitalometro, sin embargo presento respuesta positiva a la palpación y percusión. La radiografía periapical mostró un tratamiento endodóntico defectuoso, siendo que en la raíz mesial presentaba una lesión periapical de aproximadamente $3.5 \mathrm{~mm}$ de diámetro, la raíz distal ensanchamiento del espacio del ligamento periodontal (Figura 1). Al observar más detenidamente se evidencia una raíz adicional distolingual la cual también presenta lesión periapical. Posteriormente se decide realizar radiografías anguladas, en las cuales se 
diagnostica la presencia de Radix Entomolaris. (Figura 2). En la raíz disto lingual se observa la presencia de un endoposte metálico tipo "rosca". El diagnostico fue Tratamiento Previo, el diagnostico periapical fue Periodontitis Apical Aguda y el tratamiento indicado fue el Re-Tratamiento de los Conductos Radiculares.

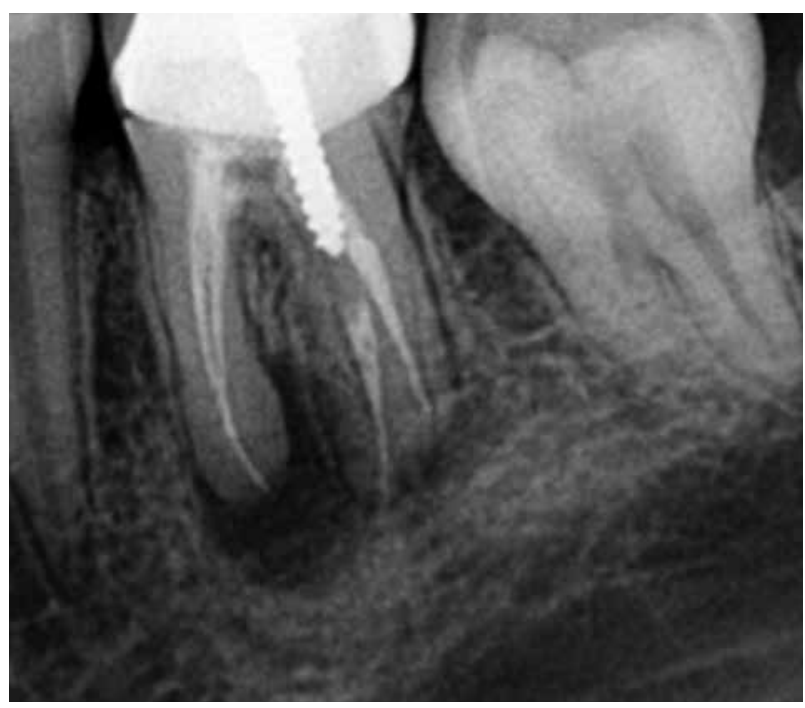

Figura 1. Radiografía Inicial.
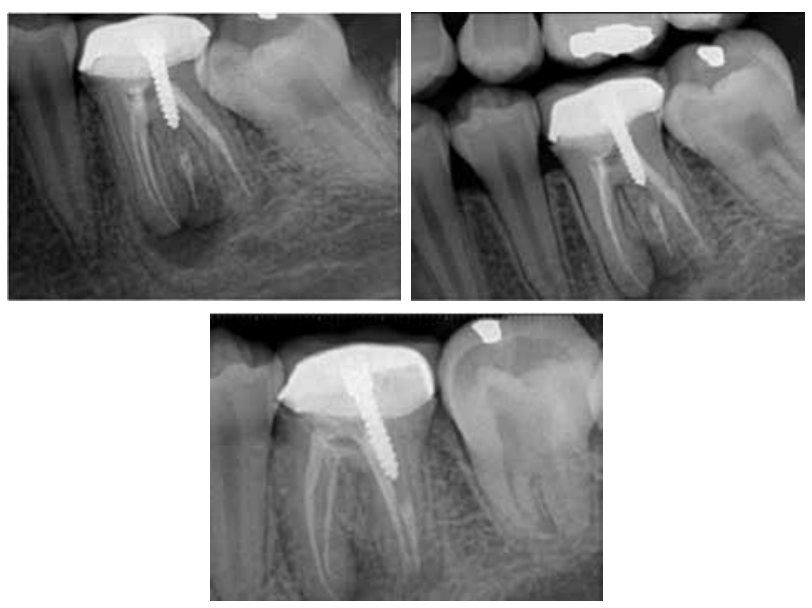

Figura 2. Radiografías Iníciales con diferentes angulaciones. Observe la presencia nítida del radix entomolaris.

A pesar de que se le insistió al paciente que sería necesario remover la corona, el mismo se negó y luego de firmar el consentimiento informado se procedió a iniciar el tratamiento. Inicialmente se realizó el aislamiento absoluto y la apertura endodóntica hasta confirmar la presencia de los cuatro conductos en la cámara pulpar, con la ayuda de una broca trans-metal acoplada a pieza de alta rotación. Al ampliar la apertura de forma trapezoidal, con el fin de buscar el radix entomolaris se observo que el endoposte se encontraba con movilidad y previo uso de ultrasónico, con ayuda de pinzas mosquito se realizo la extracción del mismo. Posteriormente se realiza la desinfección de la cámara pulpar con hipoclorito de sodio ( $\mathrm{NaOCl}$ $5 \%$ ). Con la ayuda de limas Hedstrom (Dentsply, Maillerfer, Suiza) se realizó la desobturación de los conductos radiculares. Luego se procedió a retomar y realizar la medición de longitud total de los conductos radiculares con la ayuda de un localizador RootZX II (Morita, Japón) (Figura 3). Una vez ya delimitada la longitud de trabajo, bajo copiosa irrigación de los conductos radiculares con mínimo $5 \mathrm{ml}$ de $\mathrm{NaOCl} 5 \%$ por conducto se realizo

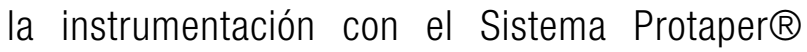
Manual (Dentsply, Maillerfer, Suiza) hasta trabajar en todos los conductos hasta una F4 (\#40). Entre cada instrumento rotatorio se realizó la patencia con una lima-k \#15 (Dentsply, Maillerfer, Suiza). Una vez finalizada la instrumentación se procedió a irrigar con $3 \mathrm{ml}$ EDTA 17\% por conducto, seguido de una irrigación final con $3 \mathrm{ml}$ de $\mathrm{NaOCl} 5 \%$. Al observar la presencia de exudado en apical de los conductos se decide colocar hidróxido de calcio como medicación intraconducto durante 8 días (ULTRACAL, Ultradent, E.E..U.U). La apertura endodóntica se obtura provisionalmente con Cavit $\circledast$ ( 3M ESPE, E.E.U.U).

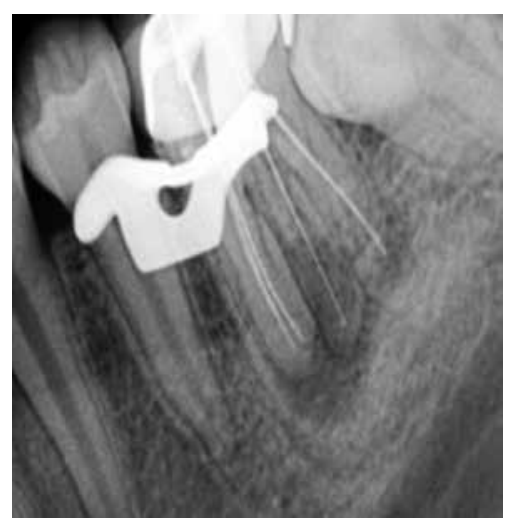

Figura 3. Radiografía de Longitud de los conductos radiculares. 
En la segunda cita el paciente refiere un total alivio a la hora de masticar. Luego se coloca nuevamente el aislamiento absoluto con dique de goma y se remueve con pieza de alta rotación el Cavit@. Con la ayuda de una lima Flexofile \#40 (Dentsply, Maillefer, Suiza) y con abundante irrigación con $\mathrm{NaOCI}$ \% se remueve la medicación intraconducto. Posteriormente se hace un lavado final con $3 \mathrm{ml}$ de EDTA y se secan los conductos con puntas de papel estéril (Dentsply, Maillefer, Suiza). Luego de desinfectar los conos principales de gutapercha se procede a realizar la radiografía y confirmar la longitud de trabajo (Figura 4). Al observarse una correcta longitud y conformación de los conductos, se procede a la obturación con la técnica de compactación lateral utilizando el cemento ROEKO ${ }^{\circ}$ (Coltene/Whaledent, E.E.U.U). Posteriormente se decide obturar la cavidad coronaria con MiracleMix ${ }^{\circledR}$ (GC, E.E.U.U) (Figura 5). Pasados 6 meses se realizo una radiografía de control post-operatorio donde se puede observar prácticamente la resolución de la lesión periapical.
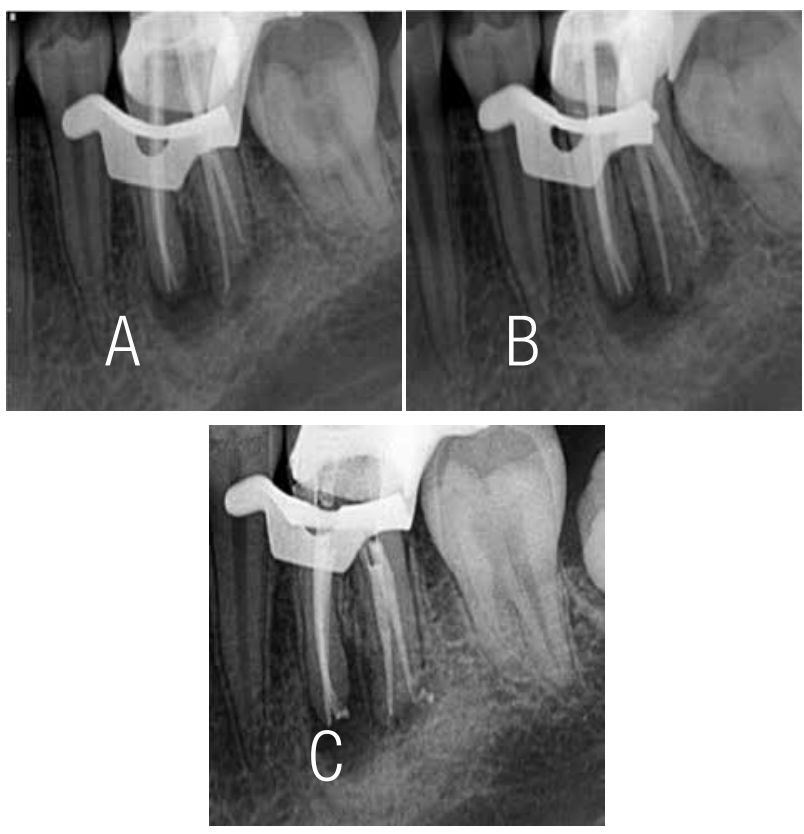

Figura 4. A y B. Radiografía Prueba de Conos. C. Radiografía para verificar la homogeneidad de la obturación de los conductos radiculares.
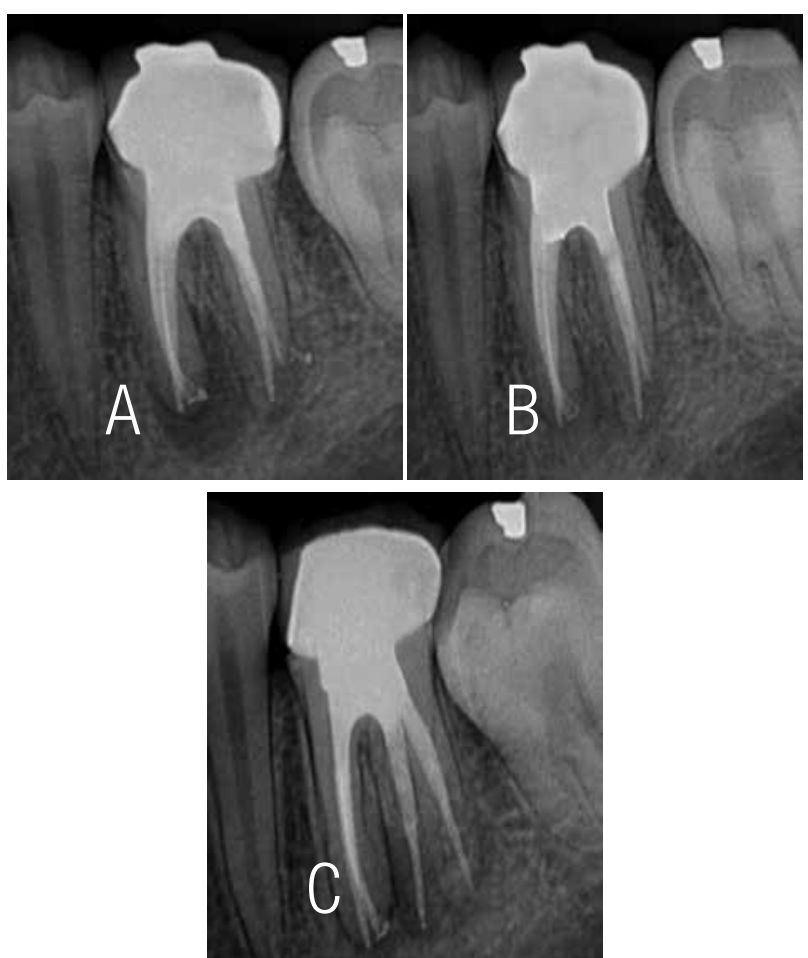

Figura 5. A. Radiografía final. B y C. Control post-operatorio. Observe el reparo óseo periapical.

\section{DISCUSIÓN}

El éxito del tratamiento del conducto radicular depende principalmente de poder cumplir con el correcto diagnostico, acceso, limpieza, conformación y obturación tridimensional de los conductos radiculares (1). El conocimiento de las variaciones morfológicas es esencial para poder cumplir con todos los aspectos.

Un caso clínico que requiera terapia endodóntica en una pieza dental con Radix Entomolaris representa un importante desafío no solo al cirujano dentista general, sino también al especialista en endodoncia; ya que su localización y posterior instrumentación se dificulta principalmente a que presentan curvaturas en el tercio apical hacia vestibular en la mayoría de los casos. Asimismo, durante el tratamiento en sí, es aconsejable 
realizar ciertas modificaciones al protocolo, como la extensión de su apertura, la cual usualmente es triangular en una molar inferior y ampliarla a trapezoidal. Esto para facilitar el ingreso en línea recta de las limas endodónticas para evitar así, accidentes como transportaciones del conducto y/ perforaciones. Esta pequeña variación favorece el establecimiento de una adecuada patencia del conducto, la cual es fundamental para promover que la solución irrigadora alcance los milímetros apicales y promueva así una apropiada desinfección del conducto radicular (1). Una adecuada desinfección y obturación de los conductos radiculares garantizan el éxito del tratamiento, con el consecuente reparo óseo a nivel periapical y ausencia de sintomatología clínica, como el observado en el presente relato clínico.

A pesar de que la presencia de un molar inferior con radix entomolaris ha sido relacionada en grupos étnicos asiáticos, indios americanos, su exacta etiología no ha sido aun elucidada. Sin embargo este tipo de casos no son poco frecuentes en el restante de la población, como lo fue en este caso clínico. Por esto el correcto diagnostico es fundamental en el desarrollo del tratamiento. Las variaciones morfológicas de los radix entomolaris se presentan en la mayoría de los casos con la inclinación de la raíz y curvatura del conducto, lo cual demanda un adecuado enfoque clínico desde la localización hasta la correcta preparación biomecánica del conducto para garantizar el éxito de la terapia endodóntica.

\section{CONCLUSIÓN}

El conocimiento de las variaciones anatómicas de las piezas dentales, en conjunto con un adecuado protocolo endodóntico es crucial para el profesional. El diagnostico radiográfico inicial del Radix Entomolaris es fundamental para establecer estrategias y las consecuentes modificaciones al protocolo que garanticen el éxito de la terapia endodóntica. 


\section{REFERENCIAS}

1. Soares Goldberg, Endodoncia, Técnica y Fundamentos, Ed. Medica panamericana, 2002, pag. $57-59,68-100,127-151$, $290-311$.

2. Carabelli G. Systematisches Handbuch DER Zahnheikunde, 2nd ed. Vienna: Braumuller and Seidel, 1844; 114.

3. Calberson FL, De Moor RJ, Deroose CA. The radix entomolaris and paramolaris: clinical approach in endodontics. J Endod. 2007 Jan;33(1):58-63. 2006 Jul 26.
4. Sperber GH, Moreau JL. Study of the number of roots and canals in Senegalese first permanent mandibular molars. Int Endod J 1998;31:112-6.

5. Yew SC, Chan K. A retrospective study of endodontically treated mandibular first molars in a Chinese population. JEndod 1993;19:471-3.

6. Curzon ME. Three-rooted mandibular permanent molars in English Caucasians. J Dent Res 1973;52:181.

7. FerrazJA, Pecora JD. Three-rooted mandibular molars in patients of Mongolian, Caucasian and Negro origin. Braz Dent J 1993;3:113-7. 